\title{
Tuberculosis Presenting as Multiple Vein Thrombosis in a Hospitalized Man
}

\author{
Neuza Soares', Mariana Pacheco1, João Rocha1, Diogo José M. Lopes², Rodrigo Correia³, \\ Inês Silva ${ }^{1}$ \\ ${ }^{1}$ Internal Medicine Department, Centro Hospitalar Universitário de SãoJoão, Porto, Portugal \\ ${ }^{2}$ Clinical Pharmacology Unit, Centro Hospitalar Universitário de SãoJoão, Porto, Portugal \\ ${ }^{3}$ Physical Medicine and Rehabilitation Deparment, Centro Hospitalar Vila Nova de Gaia/Espinho, Gaia, Portugal \\ Email: neuzasoares13@hotmail.com
}

How to cite this paper: Soares, N., Pacheco, M., Rocha, J., Lopes, D.J.M., Correia, R. and Silva, I. (2020) Tuberculosis Presenting as Multiple Vein Thrombosis in a Hospitalized Man. Journal of Tuberculosis Research, 8, 177-180.

https://doi.org/10.4236/jtr.2020.83016

Received: August 12, 2020

Accepted: September 26, 2020

Published: September 29, 2020

Copyright $\odot 2020$ by author(s) and Scientific Research Publishing Inc. This work is licensed under the Creative Commons Attribution International License (CC BY 4.0).

http://creativecommons.org/licenses/by/4.0/

\begin{abstract}
Tuberculosis (TB) remains an infectious disease with a high prevalence worldwide and represents a major public health issue. Although venous thromboembolism (VTE) is a rare complication of this disease, it may be a potentially life-threatening event. A 58-year-old man was admitted due to hematemesis due to inflammation at the anastomosis site after a gastrectomy years ago. After 3 days in-hospital, he showed a peroneal deep vein thrombosis and superficial thrombosis of left cephalic vein. Although reduced mobility and lack of prophylactic heparin could explain vein thrombosis, a simple etiologic workup was performed and active tuberculosis was diagnosed. This case illustrates a rare and unusual presentation form of tuberculosis, a condition that remains now-a-days one of the leading infectious causes of death worldwide. The association between tuberculosis and VTE is rare, but it should be systematically investigated.
\end{abstract}

\section{Keywords}

Pulmonary Tuberculosis, Thrombosis, Hypercoagulable State

\section{Introduction}

Tuberculosis (TB) is defined as an infectious disease caused by Mycobacterium tuberculosis. TB remains an infectious disease with a high prevalence worldwide and represents a major public health issue. Globally, an estimated 10.0 million (range, 9.0 - 11.1 million) people fell ill with TB in 2018. Geographically, most TB cases in 2018 were in the World Health Organization regions of South-East Asia (44\%), Africa (24\%) and the Western Pacific (18\%), with smaller percentages in the Eastern Mediterranean (8\%), the Americas (3\%) and Europe (3\%) 
[1]. TB leads to diverse symptoms and signs according to infected organs. The lungs are involved in approximately $90 \%$ of cases, while the gastrointestinal tract, genitourinary tract, lymph nodes, bone, muscle, and central nervous system can also be involved in so-called extrapulmonary tuberculosis. In addition, it is well known that a hypercoagulable state associated with tuberculosis may provoke thromboembolism [2].

Although venous thromboembolism (VTE) is a rare complication of this disease, it may be a potentially life-threatening event. VTE is a group of noncommunicable diseases or clinical syndromes caused by thromboembolism blockage of an artery or its branches, which is already categorized as the major public health problem, with high health costs and high mortality [2].

Actually, VTE can be the presenting feature of TB and occurs a few days after the diagnosis or late in the course of the disease, even in patients on anti-tuberculosis treatment. Deep venous thrombosis has been associated with $1.5 \%-3.4 \%$ cases of TB [2].

Our case showed that VTE may complicate pulmonary TB.

\section{Case Report}

A 58-year-old man with history of gastrectomy years ago due to gastric ulcer was admitted due to hematemesis. Upper endoscopy showed inflammation at the site of Billroth II anastomosis. This inflammation was thought to be responsible, proton-pump inhibitors were initiated. The patient always remained asymptomatic, hemodynamically stable, with no futher episodes of hematemesis and with no need for blood transfusions.

After 3 days in-hospital, he showed erythema and pain along the course of a hardened left cephalic vein, from arm to forearm, where he had previously had peripheral vein catheters. One day later, he presented with left leg swelling and pain. Ultrasound confirmed peroneal deep vein thrombosis and superficial thrombosis of left cephalic vein.

Although reduced mobility and lack of prophylactic heparin could explain vein thrombosis, a simple etiologic workup was performed. Complete blood count, serum chemistry and coagulation tests were unremarkable, except for normocytic and normochromic anemia ( $\mathrm{Hg} 9.6 \mathrm{~g} / \mathrm{dL}$ ). Chest radiograph (Figure 1(A)) showed multiple ill-defined nodules on apical segments of both lungs with potential area of cavitation (arrow). Chest CT scan showed pulmonary consolidation, predominantly in the apical segments, centrilobular nodules, cavitation lesions (arrow), tree-in-bud sign (red circle) and ground glass infiltrates (yellow circle), as well as mediastinal and hilaradenopathies.

Ziehl-Neelsen stain on a sputum smear revealed multiple acid-fast bacilli and treatment was started with first-line antituberculous agents. Mycobacterium tuberculosis was then confirmed on mycobacterial culture. The patient was HIV negative. Although he was asymptomatic at first, he started denoting abnormal fatigue, exertional dyspnea and hemoptoic sputum during the first weeks of treatment. 

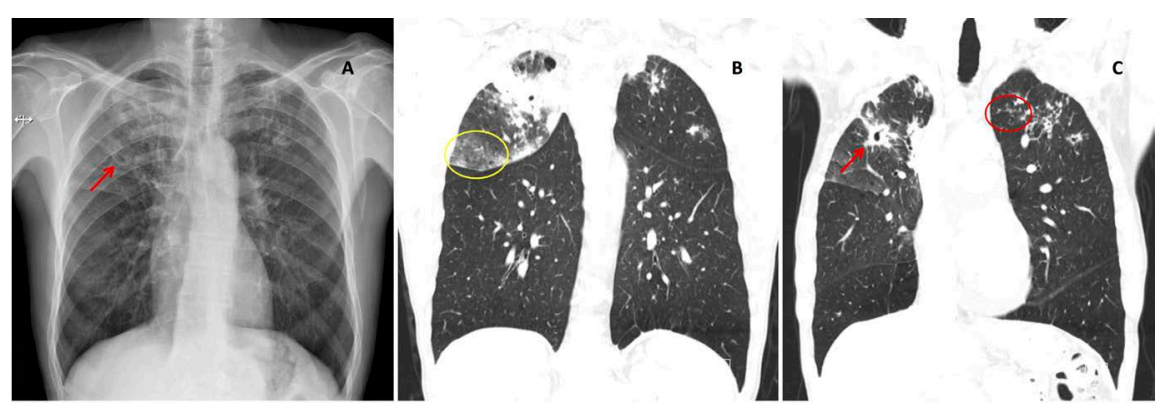

Figure 1. (A) Chest radiograph showing multiple ill-defined nodules on apical segments with potential areas of cavitation (arrow); (B) Heterogeneous consolidation involving the apical and anterior segments of the right upper lobe surrounded by ground glass infiltrates (yellow circle) on CT scan; (C) Chest CT scan showing thick walled cavitary lesion on right upper lobe (arrow) and branching linear structures (tree-in-bud sign) on left upper lobe (red circle).

\section{Discussion}

VTE is frequent among hospitalized patients, especially in the case of longer hospital stay or if other risk factors are present, such as infection [3]. Approximately one-third of patients with symptomatic VTE manifest pulmonary embolism (PE), whereas two-thirds manifest deep venous thrombosis (DVT) alone. DVT is commonly seen in postoperative patients and in patients who are admitted to the intensive care unit for a prolonged period. Its association with TB is a rare occurrence, and very few cases have been reported in literature [4].

Active tuberculosis appears to confer a risk for VTE that is similar to that reported for malignant neoplasia, and in-hospital mortality is known to be higher in patients with both conditions, comparing to those with only active tuberculosis or only VTE [4].

This case illustrates a rare and unusual presentation form of tuberculosis, a condition that remains now-a-days one of the leading infectious causes of death worldwide. Actually, tuberculosis is a disease with a wide variety of clinical presentations. Like other infectious diseases, TB can cause thrombosis by various mechanisms such as local invasion, venous compression or by producing a transitory hypercoagulable state. While the mechanism responsible for development, deep vein thrombosis in tuberculosis is unclear. Recent studies have established a link between haemostatic changes and this prothrombotic phase, and have demonstrated that these can normalize with an adequate antitubercular therapy (ATT). Early initiation of ATT treatment along with anticoagulant therapy decreases the overall morbidity and mortality associated with the disease [5].

Physicians should be aware of this phenomenon in order to ensure an early diagnosis and a prompt treatment, preventing fatal outcomes and publics' health threat.

\section{Conflicts of Interest}

The authors declare no conflicts of interest regarding the publication of this paper. 


\section{References}

[1] World Health Organization (2019) Global Tuberculosis Report 2019.

[2] White, N.W. (1989) Venous Thrombosis and Rifampicin. Lancet, 2, 434-435. https://doi.org/10.1016/S0140-6736(89)90603-X

[3] Rogers, M.A., Levine, D.A., Blumberg, N., Flanders, S.A., Chopra, V. and Langa, K.M. (2012) Triggers of Hospitalization for Venous Thromboembolism. Circulation, 125, 2092-2099. https://doi.org/10.1161/CIRCULATIONAHA.111.084467

[4] Gupta, A., Mrigpuri, P., Faye, A., Bandyopadhyay, D. and Singla, R. (2017) Pulmonary Tuberculosis: An Emerging Risk Factor for Venous Thromboembolism: A Case Series and Review of Literature. Lung India, 34, 65-69.

https://doi.org/10.4103/0970-2113.197110

[5] Gonçalves, I.M., Alves, D.C., Carvalho, A., Brito, M.C., Calvario, F. and Duarte, R. (2009) Tuberculosis and Venous Thromboembolism: A Case Series. Cases Journal, 2, 9333. https://doi.org/10.1186/1757-1626-2-9333 